\title{
Tidal stripping and disk kinematics in the RW Aurigae system ${ }^{\star}, \star \star$
}

\author{
S. Cabrit ${ }^{1}$, J. Pety ${ }^{1,2}$, N. Pesenti ${ }^{3}$, and C. Dougados ${ }^{3}$ \\ 1 LERMA, UMR 8112 du CNRS, Observatoire de Paris, 61 Av. de l'Observatoire, 75014 Paris, France \\ e-mail: sylvie.cabrit@obspm.fr \\ 2 IRAM, 300 rue de la Piscine, 38406 Grenoble Cedex, France \\ e-mail: pety@iram.fr \\ ${ }^{3}$ Laboratoire d'Astrophysique, UMR 5571 du CNRS, Observatoire de Grenoble, France \\ e-mail: [Nicolas.Pesenti; Catherine.Dougados]@obs.ujf-grenoble.fr
}

Received 14 August 2005 / Accepted 6 March 2006

\section{ABSTRACT}

\begin{abstract}
We present interferometric maps of the RW Aur system obtained with the IRAM Plateau de Bure Interferometer in $\mathrm{CO}(J=2-1)$, $\mathrm{CO}(J=1-0)$, and nearby continuum. The sub-arcsecond angular resolution $\left(0.89^{\prime \prime} \times 0.58^{\prime \prime}\right)$ and high-sensitivity reached at $1.3 \mathrm{~mm}$ enable us to resolve three molecular structures: (1) an optically thick disk around RW Aur A in rotation about the optical jet axis, (2) a disturbed asymmetric peak around RW Aur B, (3) a 600 AU-long "arm" of material trailing from the RW Aur A disk. Comparison with Keplerian models indicates that the RW Aur A disk is the smallest detected so far around a T Tauri star (radius 40-57 AU) and that the CO emitting layer at the outer edge is warmer than the dust $\left(T_{\text {out }} \simeq 60-100 \mathrm{~K}\right)$ and relatively thick $\left(N_{\text {warm }} \simeq 0.1-10 \times 10^{22} \mathrm{~cm}^{-2}\right)$. The morphology and kinematics of the detected features strongly suggest that we are witnessing tidal stripping of the primary disk by the recent fly-by of RW Aur B. We speculate that tidal dissipation might explain the warmer gas temperatures in the RW Aur A disk compared with typical T Tauri stars, and perhaps play a role in its elevated accretion rate. We also find that the rotation sense of the RW Aur A disk is opposite to transverse velocity shifts in the optical jet reported by Woitas et al. (2005, A\&A, 432, 149). We argue that these transverse shifts are likely to represent only upper limits to the true jet rotation speed. The limits remain consistent with current models of MHD launching from the disk.
\end{abstract}

Key words. stars: individual: RW Aur - stars: formation - stars: circumstellar matter - ISM: molecules - radio lines: ISM

\section{Introduction}

The young RW Aur system in Taurus-Auriga is composed of two actively accreting "Classical" $T$ Tauri stars (hereafter denoted CTTS): an early-K star, RW Aur A, and a 2-3 mag fainter secondary located 1.5" away, RW Aur B (Herbig \& Bell 1988; Duchêne et al. 1999; Stout-Batalha et al. 2000). A third, faint $K$-band source denoted RW Aur C was reported 0.12" from B (Ghez et al. 1993), but was not confirmed by subsequent HST optical imaging (Ghez et al. 1997; White \& Ghez 2001). Resolved photometry and recent evolutionary tracks indicate masses of 1.3-1.4 $M_{\odot}$ for RW Aur A and 0.7-0.9 $M_{\odot}$ for RW Aur B (Ghez et al. 1997; Woitas et al. 2001) at a distance $d=140 \mathrm{pc}$ (Bertout et al. 1999).

RW Aur A has attracted particular attention as it exhibits one of the highest accretion rates among CTTS $(\simeq 2-10 \times$ $10^{-7} M_{\odot} \mathrm{yr}^{-1}$, Basri \& Bertout 1989; Hartigan et al. 1995), i.e. 10-100 times higher than typical. It also drives one of the brightest atomic jets in Taurus-Auriga, topped only by DG Tau and HL Tau (Hirth et al. 1994; Mundt \& Eislöffel 1998; Dougados et al. 2000; Woitas et al. 2002). RW Aur A is further peculiar for showing large variations in radial velocity and emission line properties over a 2.77 day period, attributed to a close

\footnotetext{
* Based on observations obtained with the IRAM Plateau de Bure interferometer. IRAM is supported by CNRS (France), MPG (Germany), and IGN (Spain).

$\star \star$ Figure A.1 is only available in electronic form at http://www.edpsciences.org
}

low-mass companion or to a rotationally modulated accretion "hot spot" (Gahm et al. 1999; Petrov et al. 2001).

There are several important motivations to study the circumstellar environment of the RW Aur system at high angular resolution:

First, unlike other CTTS of comparable high accretion rates, RW Aur A appears to have a particularly low disk mass $\simeq 3 \times$ $10^{-4} M_{\odot}$ from single-dish sub/mm continuum measurements (Osterloh \& Beckwith 1995; Andrews \& Williams 2005), and lies in a region devoid of large-scale $\mathrm{CO}$ emission (Ungerechts \& Thaddeus 1987). Hence, one faces an acute problem to replenish the disk and to sustain steady accretion at the current rate beyond a thousand years, unless a hidden mass reservoir is present on circumstellar scales.

Second, the RW Aur A jet is one of the four CTTS jets where transverse velocity shifts have been recently detected with STIS/HST. If these shifts are due to jet rotation, they would imply that the optical jets are magnetically launched from the disk surface at relatively large radii of $0.2-3 \mathrm{AU}$, thus solving the long-lasting question of the jet origin in CTTS (Bacciotti et al. 2002; Anderson et al. 2003; Coffey et al. 2004; Woitas et al. 2005). To support this interpretation, however, it is crucial to check that the underlying disk rotates in the same sense as the jet, as verified so far only in the DG Tau case (Testi et al. 2002).

Finally, if CTTS jets do trace magnetized disk winds, a molecular counterpart to the atomic optical jet will be present if MHD ejection operates beyond a few AU (Safier 1993). The lack of ambient molecular cloud toward RW Aur A means that any detected molecular flow in this system would necessarily be 
Table 1. Observation parameters.

\begin{tabular}{|c|c|c|c|c|c|c|c|c|}
\hline Phase center & $\overline{\alpha_{2000}=05^{\mathrm{h}} 07^{\mathrm{m}}}$ & $\overline{\delta_{2000}=}$ & $30^{\circ} 24^{\prime}$ & $05.16^{\prime \prime}$ & & & & \\
\hline Molecule \& line & $\begin{array}{c}\text { Frequency } \\
\mathrm{GHz}\end{array}$ & $\begin{array}{c}F W H M \text { Beam } \\
\text { arcsec }\end{array}$ & $\begin{array}{c}\mathrm{PA} \\
\circ\end{array}$ & $\begin{array}{c}\Delta v^{a} \\
\mathrm{~km} \mathrm{~s}^{-1}\end{array}$ & $\begin{array}{l}\mathrm{rms} \mathrm{noise}^{a} \\
\mathrm{mJy} / \text { Beam }^{2}\end{array}$ & $\begin{array}{c}\begin{array}{c}\text { Int. time } \\
\text { hours }\end{array} \\
\end{array}$ & Obs. date & $\begin{array}{c}\text { Flux scale } \\
\mathrm{K} /(\mathrm{Jy} / \text { Beam })\end{array}$ \\
\hline $\mathrm{C}^{18} \mathrm{O} J=1-0$ & 109.782 & $2.57 \times 1.60$ & 41 & 0.85 & 7 & 7.2 & $2002-2003$ & 24.7 \\
\hline${ }^{13} \mathrm{CO} \mathrm{J}=1-0$ & 110.201 & $2.56 \times 1.59$ & 41 & 0.85 & 7 & 7.2 & 2002-2003 & 24.7 \\
\hline${ }^{12} \mathrm{CO} J=1-0$ & 115.271 & $2.42 \times 1.55$ & 24 & 0.81 & 10 & 9.7 & 2003-2004 & 24.5 \\
\hline${ }^{12} \mathrm{CO} J=2-1$ & 230.538 & $0.89 \times 0.58$ & 23 & 0.41 & 18 & 16.9 & 2002-2004 & 44.5 \\
\hline $\begin{array}{c}\text { Continuum } \lambda \\
\mathrm{mm}\end{array}$ & $\begin{array}{c}\text { Frequency } \\
\mathrm{GHz}\end{array}$ & $\begin{array}{c}F W H M \text { Beam } \\
\text { arcsec }\end{array}$ & $\overline{\mathrm{PA}}$ & $\begin{array}{c}\Delta v \\
\mathrm{MHz}\end{array}$ & $\begin{array}{l}\mathrm{rms} \text { noise } \\
\mathrm{mJy} / \text { Beam }\end{array}$ & $\begin{array}{l}\text { Int. time } \\
\text { hours }\end{array}$ & Obs. date & \\
\hline $2.66^{b}$ & $112.5^{b}$ & $1.84 \times 1.22^{b}$ & 31 & $580^{c}$ & 0.24 & 16.9 & $2002-2004$ & \\
\hline 1.30 & 230.5 & $0.87 \times 0.56$ & 22 & $1160^{c}$ & 0.47 & 16.9 & $2002-2004$ & \\
\hline
\end{tabular}

${ }^{a} \Delta v$ and the rms noise refer to the rebinned resolution in the channel maps. The native correlator resolution is 8 times higher.

${ }^{b}$ Merge of the 2.6 and $2.7 \mathrm{~mm}$ measurements.

${ }^{c}$ Total IF bandwidth currently available at PdBI (with SSB tuning at $2.6 \mathrm{~mm}$ and DSB at $1.3 \mathrm{~mm}$ ).

ejected and not entrained material: hence, such an observation would provide independent, direct evidence for extended disk winds in CTTS.

To address these issues, we have undertaken an interferometric mapping program of the RW Aur system at the Plateau de Bure IRAM Interferometer in CO isotopologues at $1.3 \mathrm{~mm}$ and $2.6 \mathrm{~mm}$, and in the nearby continuum. We detail our observations and data reduction in Sect. 2, and describe the new features detected in our maps and their kinematics in Sect. 3. In Sect. 4, we discuss their physical properties and the resulting implications. We summarize our conclusions in Sect. 5.

\section{Observations and data reduction}

The characteristics of our line and continuum observations are detailed below. The resulting beam sizes and sensitivities are summarized in Table 1.

\section{1. ${ }^{13} \mathrm{CO} \mathrm{J}=1-0$ and $\mathrm{C}^{18} \mathrm{O} \mathrm{J}=1-0$}

The first PdBI observations dedicated to this project were carried out with 6 antennas in the $\mathrm{B}$ and $\mathrm{C}$ configurations (baseline lengths from 48 to $331 \mathrm{~m}$ ) during the winter 2002/2003. The $\mathrm{C}^{18} \mathrm{O} J=1-0$ and ${ }^{13} \mathrm{CO} J=1-0$ lines were observed simultaneously using the $3 \mathrm{~mm}$ receivers (tuned single sideband at $110.1 \mathrm{GHz}$ ). Two correlator bands of $20 \mathrm{MHz}$ were respectively centered on the ${ }^{13} \mathrm{CO} J=1-0$ and $\mathrm{C}^{18} \mathrm{O} J=1-0$. The total telescope time, including phase and amplitude calibrators and tuning, amounts to $18 \mathrm{~h}$. In practice, only data with phase noise better than $30^{\circ}$ were used. The on-source integration time of useful data is then $7.2 \mathrm{~h}$.

\section{2. ${ }^{12} \mathrm{CO} \mathrm{J}=1-0$}

As a follow-up, we carried out observations of ${ }^{12} \mathrm{CO}(J=1-0)$ at PdBI with 6 antennas in the A, C and D configurations (baseline lengths from 24 to $400 \mathrm{~m}$ ) during the winter 2003/2004. The $3 \mathrm{~mm}$ receivers were tuned single sideband at $115.271 \mathrm{GHz}$. One correlator band of $20 \mathrm{MHz}$ was centered on the ${ }^{12} \mathrm{CO} J=1-0$. The total telescope time amounts to $32 \mathrm{~h}$ with 6 antennas. This leads to on-source integration time of useful data of $9.7 \mathrm{~h}$ after filtering out data with phase noise worse than $30^{\circ}$.

\section{3. $2.66 \mathrm{~mm}$ continuum}

Two bands of $320 \mathrm{MHz}$ were used to measure the $2.7 \mathrm{~mm}$ continuum during the winter 2002/2003 and the $2.6 \mathrm{~mm}$ continuum during the winter $2003 / 2004$. To improve signal-to-noise ratio and $u v$ coverage, we merged both data sets to obtain an "equivalent" $2.66 \mathrm{~mm}$ continuum image. No color correction was applied, as it would have been smaller than the precision of the flux calibration. The total on-source integration time of the useful data is thus the sum of the previous two integration time, i.e. $16.9 \mathrm{~h}$.

\section{4. ${ }^{12} \mathrm{CO} \mathrm{J}=2-1$ and $1.3 \mathrm{~mm}$ continuum}

During both winters, we used the dual frequency possibility of PdBI to observe the ${ }^{12} \mathrm{CO} J=2-1$ line and $1.3 \mathrm{~mm}$ continuum in parallel to the $2.66 \mathrm{~mm}$ observations. One correlator band of $20 \mathrm{MHz}$ was centered on the ${ }^{12} \mathrm{CO} J=2-1$ and two bands of $320 \mathrm{MHz}$ were used for the $1.3 \mathrm{~mm}$ continuum. The line and continuum were thus observed with 6 antennas in all configurations (A to D) available at PdBI. The total telescope time amounts to $50 \mathrm{~h}$. Only data with phase noise better than $60^{\circ}$ were used. The remaining on-source integration time of the useful data is $16.9 \mathrm{~h}$. This results in sub-arcsecond resolution (FWHM beam of $\left.0.89^{\prime \prime} \times 0.58^{\prime \prime}\right)$ at high signal-to-noise ratio (cf. Table 1$)$.

\subsection{Data reduction}

All data were reduced using the GILDAS ${ }^{1}$ software. Standard calibration methods using close calibrators were applied to all the PdBI data. Calibrator fluxes used for absolute flux calibration are summarized in Table 2. The J0418+380 and B0528+134 calibrators were both observed along with MWC349 (used as primary flux calibrator) during each observing session. An available model of the MWC349 flux as a function of frequency (time variations are negligible) was then used to bootstrap the fluxes of $\mathrm{J} 0418+380$ and $\mathrm{B} 0528+134$ as a function of time and frequency. As a cross-check, we compared the B0528+134 fluxes obtained here with measures obtained at PdBI during the same time period using other primary flux calibrators, i.e. the continuum of LkCa15 and MWC480 (Dutrey \& Piétu, private communication). This comparison leads us to estimate an absolute flux accuracy of $20 \%$.

\footnotetext{
${ }^{1}$ See http://www.iram.fr/IRAMFR/GILDAS for more information about the GILDAS software.
} 
Table 2. Calibrator fluxes in Jy (bootstrapped using the MWC349 flux model, see text).

\begin{tabular}{lrrrr}
\hline \hline & \multicolumn{2}{c}{ B0528+134 } & \multicolumn{2}{c}{ J0418+380 } \\
& $2.66 \mathrm{~mm}$ & $1.3 \mathrm{~mm}$ & $2.66 \mathrm{~mm}$ & $1.3 \mathrm{~mm}$ \\
\hline 25.12 .2002 & 1.9 & 1.5 & 3.1 & 2.5 \\
18.01 .2003 & 1.9 & 1.5 & 3.1 & 2.2 \\
08.02 .2003 & 1.9 & 1.5 & 2.7 & 1.9 \\
01.03 .2004 & 1.9 & 1.5 & 1.9 & 1.2 \\
16.03 .2004 & 1.9 & 1.5 & 2.0 & 1.2 \\
05.04 .2004 & 1.9 & 1.5 & 2.0 & 1.2 \\
\hline
\end{tabular}

Low quality data were filtered out based on the pointing, focus and atmospheric phase noise measurements. ${ }^{12} \mathrm{CO} J=1-0$, ${ }^{13} \mathrm{CO} J=1-0$ and $\mathrm{C}^{18} \mathrm{O} J=1-0$ images were produced using natural weighting of the visibilities to get the highest possible signal-to-noise ratio. The ${ }^{12} \mathrm{CO} J=2-1$ and $2.66 \mathrm{~mm}$ and $1.3 \mathrm{~mm}$ continuum images were produced using robust weighting, to get the highest possible resolution at the cost of a $10 \%$ reduction of the point source sensitivity. Note that all velocities are expressed in the heliocentric rest-frame, to ease direct comparison with optical data.

\section{Results}

\subsection{Continuum emission}

We resolve for the first time the continuum emission from the RW Aur binary system, as illustrated in Panel (a) of Fig. 1 at $1.3 \mathrm{~mm}$. A fit to the $u-v$ plane data was carried out both at $1.3 \mathrm{~mm}$ and $2.6 \mathrm{~mm}$ to determine more precisely the individual continuum source fluxes and positions. Results are given in Table 3.

The fitted position for the main peak shows a significant offset $\Delta \delta=-0.067^{\prime \prime} \pm 0.007^{\prime \prime}$ from the ICRS 2000 coordinates of RW Aur A (our adopted phase center). This offset is consistent with the stellar proper motion of $\mu_{\alpha}=3.26 \pm 3.44$ mas $\mathrm{yr}^{-1}$,

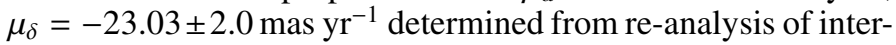
mediate astrometric Hipparcos data (Bertout et al. 1999), given our observing date range (2002-2004). Hence, the main continuum peak coincides with RW Aur A.

Our fit gives a separation between RW Aur A and the secondary continuum peak of $1.468^{\prime \prime} \pm 0.056$ at $\mathrm{PA}=255^{\circ} .3$, consistent with determination of the $\mathrm{AB}$ separation in the optical with HST: $1.4175^{\prime \prime} \pm 0.0034^{\prime \prime}$ at PA $=255^{\circ} .459 \pm 0.065$ (Ghez et al. 1997). We thus conclude that the secondary continuum peak is centered on RW Aur B within our uncertainties.

RW Aur A is 5-10 times brighter than RW Aur B in the continuum at $1.3 \mathrm{~mm}$ and $2.6 \mathrm{~mm}$. The total $1.3 \mathrm{~mm}$ flux from the system, $32.8 \pm 0.7 \mathrm{mJy}$, is slightly lower than the value of $42 \pm 5$ mJy measured by Osterloh \& Beckwith (1995) inside a $12^{\prime \prime}$ beam $^{2}$, but the difference is only $2 \sigma$ and thus not significant.

The spectral index between $1.3 \mathrm{~mm}$ and $2.6 \mathrm{~mm}$, defined as $\alpha=\Delta \log F_{v} / \Delta \log v$, is $\alpha=1.73 \pm 0.08$ for the continuum emission of RW Aur A. A similar slope of $\alpha=1.43 \pm 0.21$ was previously found in the submm range by Andrews \& Williams (2005). We find a steeper mm slope for RW Aur B, $\alpha=2.6 \pm 0.7$.

\subsection{Line emission}

Line emission was detected only in ${ }^{12} \mathrm{CO}(J=2-1)$ and ${ }^{12} \mathrm{CO}(J=1-0) ;{ }^{13} \mathrm{CO}$ and $\mathrm{C}^{18} \mathrm{O}$ remained undetected. Channel

${ }^{2}$ The value of $421 \mathrm{mJy}$ in their table is a typographical error (Osterloh, private communication).

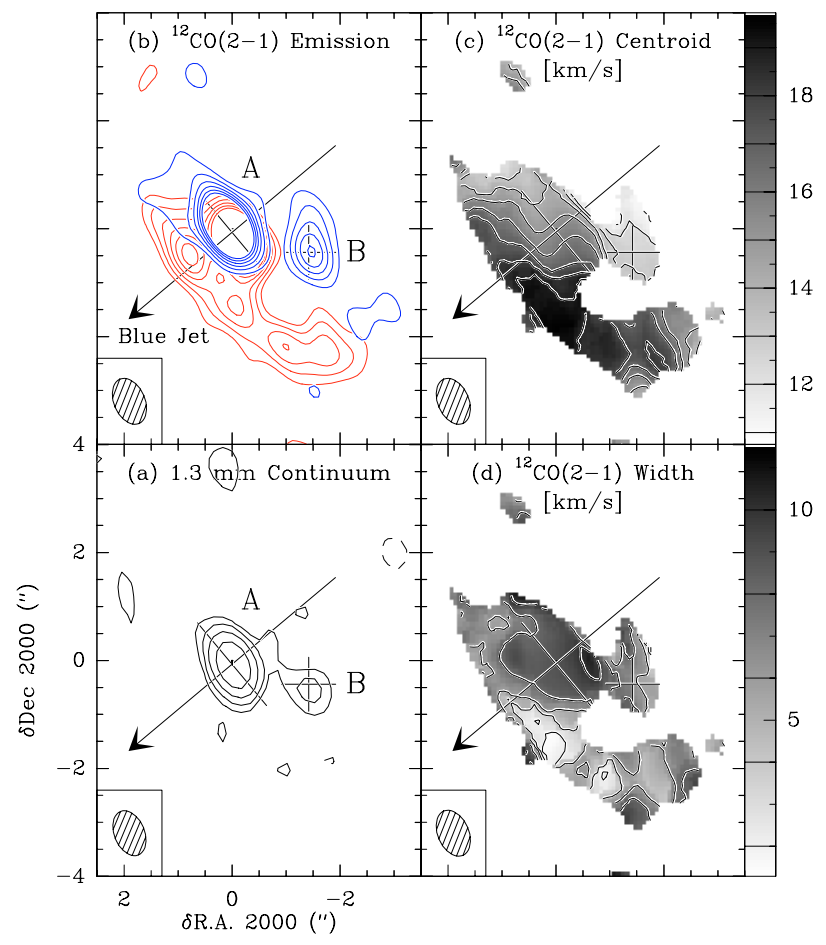

Fig. 1. a) $1.3 \mathrm{~mm}$ continuum map of the RW Aur system. Contour levels are $3,6,12,24,48 \sigma(1 \sigma=0.47 \mathrm{mJy} / \mathrm{beam})$. Crosses indicate the peak positions, which closely correspond to RW Aur A and B. The large arrow shows the direction of the blueshifted jet. b) ${ }^{12} \mathrm{CO} J=2-1$ emission blueshifted and redshifted with respect to the RW Aur A systemic velocity. Intensity was integrated from $V_{\text {helio }}=8$ to $15.5 \mathrm{~km} \mathrm{~s}^{-1}$ (dark contours) and from 15.9 to $23 \mathrm{~km} \mathrm{~s}^{-1}$ (grey contours). Contour spacing is $3 \sigma\left(0.12 \mathrm{Jy} \mathrm{km} \mathrm{s}^{-1}\right)$, truncated to $18 \sigma$ for clarity. c), d) Heliocentric centroid velocity and line width (i.e. first and second order moments) of the ${ }^{12} \mathrm{CO} J=2-1$ line. Only pixels with integrated emission higher than $4 \sigma$ are shown. (This figure is available in color in the electronic version.)

maps in ${ }^{12} \mathrm{CO}(J=2-1)$ are displayed in Fig. 2. The lower angular resolution channel maps in ${ }^{12} \mathrm{CO}(J=1-0)$ are presented in Fig. A.1 (on-line material only).

The key features detected in ${ }^{12} \mathrm{CO}(J=2-1)$ are illustrated in Figs. $1 \mathrm{~b}-\mathrm{d}$. Panel (b) presents maps of ${ }^{12} \mathrm{CO}(J=2-1)$ intensity, integrated separately over blue-shifted and red-shifted velocity ranges defined with respect to the RW Aur A systemic velocity $\left(V_{\text {helio }} \simeq 15.8 \mathrm{~km} \mathrm{~s}^{-1}\right.$ (see below)). Panels (c) and (d) present maps of the ${ }^{12} \mathrm{CO}(J=2-1)$ centroid velocity and line width (first and second order moments), illustrating the global kinematics. Three main structures are detected in our field of view:

1. A bright, compact structure centered on RW Aur A, with both blue- and red-shifted emission.

2. A fainter, more diffuse structure centered on RW Aur B, entirely blueshifted.

3. A large "arm" wrapping around RW Aur A, almost entirely redshifted.

No molecular jet or outflow is detected down to our sensitivity limit.

Integrated ${ }^{12} \mathrm{CO}(J=2-1)$ fluxes for each structure are given in Table 4. Below, we describe the morphology and kinematics of each structure in more detail, and we use the ${ }^{12} \mathrm{CO}(J=2-1) /(J=1-0)$ ratios and integrated intensities to estimate the line optical depth and minimum gas mass. 
Table 3. Fluxes and positions for the 2 continuum peaks inferred from global fits to the $u v$ plane data.

\begin{tabular}{cccccc}
\hline \hline Source & $\begin{array}{c}\text { Frequency } \\
(\mathrm{GHz})\end{array}$ & $\begin{array}{c}\text { Wavelength } \\
(\mathrm{mm})\end{array}$ & Fitted shape & $\begin{array}{c}\text { Flux }^{a} \\
\left(\mathrm{mJy}^{2}\right.\end{array}$ & $\begin{array}{c}\text { Offset from phase center } \\
\left({ }^{\prime \prime}\right)\end{array}$ \\
\hline RW Aur A & 230.5 & 1.30 & Gaussian & $27.6 \pm 0.5$ & $0.000 \pm 0.005 \times-0.067 \pm 0.007$ \\
& 112.5 & 2.66 & Point & $8.0 \pm 0.3$ & $-0.06 \pm 0.03 \times-0.07 \pm 0.02$ \\
\hline RW Aur B & 230.5 & 1.30 & Point & $5.2 \pm 0.4$ & $-1.42 \pm 0.03 \times-0.44 \pm 0.04$ \\
& 112.5 & 2.66 & Point & $0.8 \pm 0.3$ & $-1.34 \pm 0.28 \times-0.48 \pm 0.24$ \\
\hline
\end{tabular}

${ }^{a}$ Quoted 1- $\sigma$ flux uncertainties only reflect thermal and phase noises. The absolute flux calibration accuracy is estimated to be $20 \%$ (see text).

${ }^{b}$ Fitting an elliptical Gaussian perpendicular to the jet instead of a circular Gaussian does not significantly change the results.



Fig. 2. Channel maps of ${ }^{12} \mathrm{CO} J=2-1$ towards RW Aur, rebinned to a spectral resolution of $0.41 \mathrm{~km} \mathrm{~s}^{-1}$. Radial velocities (heliocentric) are given in the upper left corner of each panel. The large cross marks the position of RW Aur A, with an arrow pointing in the blue jet direction. The small cross marks the position of RW Aur B. Blue and red contours respectively indicate blue and red-shifted channels with respect to the RW Aur A mean systemic velocity $\left(V_{\text {helio }} \simeq 15.87 \pm 0.55 \mathrm{~km} \mathrm{~s}^{-1}\right.$, Gahm et al. 1999). Contour levels correspond to $-3,3,6$ and $12 \sigma$ with $1 \sigma=18 \mathrm{mJy} / \mathrm{Beam}=$ $0.8 \mathrm{~K}$ (see Table 1 for details). Dotted lines show negative contours. The hatched ellipse represents the beam $F W H M$ size, $0.89^{\prime \prime} \times 0.58^{\prime \prime}$ at $\mathrm{PA}=23^{\circ}$. Continuum is not subtracted. (This figure is available in color in the electronic version.)

\subsubsection{RW Aur A molecular peak}

The bright compact ${ }^{12} \mathrm{CO}$ component centered on RW Aur A has several notable properties:

(1) A centroid velocity gradient is clearly seen in Fig. 1c along $\mathrm{PA}=40^{\circ}$ (herafter the $x$ axis), i.e. exactly perpendicular to the optical jet (with blue lobe at $\mathrm{PA}=130^{\circ}$; Dougados et al. 2000; Woitas et al. 2005). Such a direction for the velocity gradient points to rotation motions, as opposed to infall or outflow.

In Fig. 3 we examine this gradient in more detail. The top panel of Fig. 3 shows that it corresponds to a small but 
Table 4. ${ }^{12} \mathrm{CO}(J=2-1)$ fluxes in $\mathrm{Jy} \mathrm{km} \mathrm{s}^{-1}$ integrated over the main structures in our map. Estimated accuracy is $20 \%$ for the largest fluxes.

\begin{tabular}{lccc}
\hline \hline Source & Red & Blue & Red+Blue \\
\hline RW Aur A & +1.7 & +1.5 & +3.2 \\
RW Aur B & +0.05 & +0.55 & +0.59 \\
Arm & +2.1 & +1.6 & +3.7 \\
Total & +4.2 & +3.3 & +7.5 \\
\hline
\end{tabular}
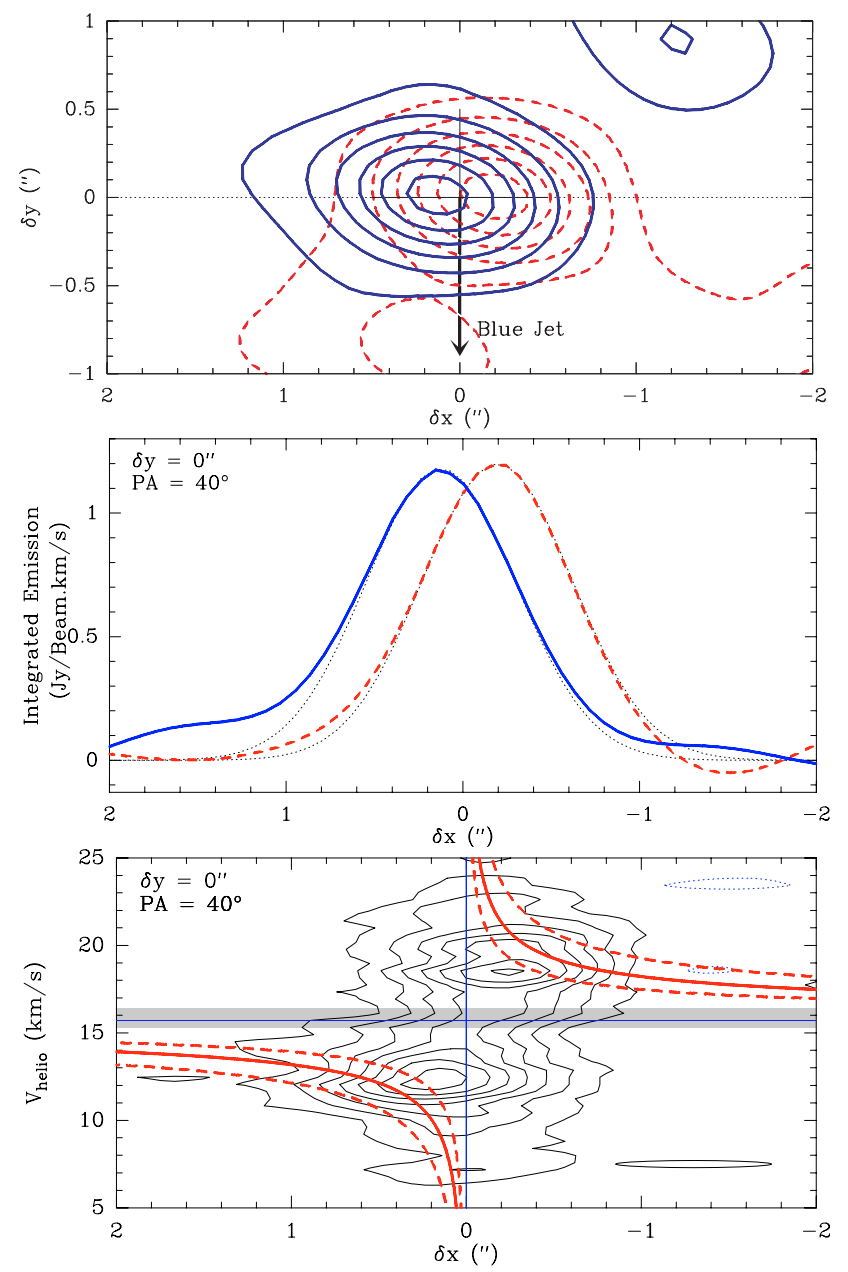

Fig. 3. Evidence for disk rotation towards RW Aur A. Top: ${ }^{12} \mathrm{CO} J=2-1$ map from Fig. 1b rotated so that the blueshifted optical jet points in the $-y$ direction. Solid contours: blueshifted emission. Dashed contours: redshifted emission. Contour spacing is $4.5 \sigma$ $\left(0.18 \mathrm{Jy} \mathrm{km} \mathrm{s}^{-1}\right)$. Middle: intensity cuts along $x$ at PA $=40^{\circ}$, with Gaussian fits of identical 1.0" FWHM overplotted. The blueshifted and redshifted emissions are centered respectively at $\delta x=0.125^{\prime \prime}$ and $\delta x=-0.195^{\prime \prime}$. Note the high signal to noise of the data. Bottom: ${ }^{12} \mathrm{CO} J=2-1$ position-velocity diagram along $x$ at $\mathrm{PA}=40^{\circ}$. Contour spacing is $2 \sigma$ (36 mJy/Beam). Horizontal and vertical lines indicate the systemic velocity range and the position of RW Aur A. Keplerian curves are drawn for $M_{\star} \times \sin i=0.5,1$ and $2 M_{\odot}$ (where $i$ is inclination from pole-on). The middle curve corresponds to typical parameters for RW Aur A (see text). The ellipse in the lower right corner illustrates the angular and velocity resolution.

definite offset along $x$ between the redshifted and blueshifted emission peaks. The middle panel of Fig. 3 plots intensity cuts along the $x$ axis, and corresponding Gaussian fits. The data quality is clearly very high, and enables us to measure with high confidence a shift of centroid positions of $0.32^{\prime \prime}$, even though it is only a third of the beam size in that direction.

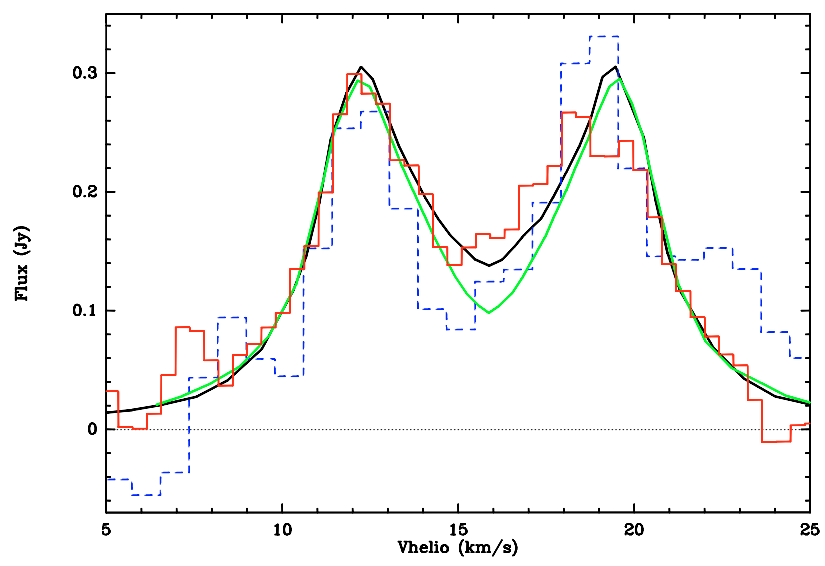

Fig. 4. ${ }^{12} \mathrm{CO}$ spectra of the RW Aur A molecular peak. Continuum emission is subtracted. The solid histogram plots the ${ }^{12} \mathrm{CO} J=2-1$ spectrum, integrated over an ellipse of size $1.8^{\prime \prime} \times 1.3^{\prime \prime}, \mathrm{PA}=40^{\circ}$. The dashed histogram shows the ${ }^{12} \mathrm{CO} J=1-0$ spectrum, multiplied by 4 (theoretical $J=2-1 / J=1-0$ flux ratio for optically thick emission in the Rayleigh-Jeans limit). The solid curves show theoretical profiles from Beckwith \& Sargent (1993) for an optically thick Keplerian disk viewed at $i=45^{\circ}$ (upper curve) and $i=60^{\circ}$ (lower curve). Inferred values of disk radius and outer temperature are discussed in Sect. 4, and listed in Table 5 .

Indeed, we are here interested only by the relative position of features in two different channels. This is a much simpler problem than absolute position accuracy because the baseline or instrumental phase uncertainties as well as uncalibrated atmospheric effects are similar for all spectral channels and cancel out in channel to channel phase differences. The remaining source of systematic uncertainty is then the precision of the phase calibration across the bandpass. In our case, the phase bandpass rms was at most $4^{\circ}$, which implies minor relative position uncertainties of $<\theta \times(4 / 360) \simeq 0.01^{\prime \prime}$ where $\theta$ is the beam size. The dominant position uncertainty thus comes from the signal-to-noise ratio $S N R$. Reid et al. (1988) show it amounts to $0.5 \theta / S N R$ or $<0.02^{\prime \prime}$ as $S N R \gtrsim 25$ for the integrated maps of RW Aur A.

(2) A position-velocity (PV) diagram along PA $=40^{\circ}$ (bottom panel of Fig. 3) shows almost perfect point-symmetry in both space and velocity with respect to $R W$ Aur A, given the mean systemic heliocentric velocity of $15.87 \pm 0.55 \mathrm{~km} \mathrm{~s}^{-1}$ derived from optical spectra by Gahm et al. (1999). This indicates a globally axisymmetric (rotating) structure around RW Aur A.

(3) The PV diagram further shows that the spatial offsets are consistent with bound motions around RW Aur A $\left(M_{\star} \simeq 1.4 \pm\right.$ $\left.0.1 M_{\odot}\right)$ : The middle solid curve plots a Keplerian curve for a disk inclination $i=45^{\circ}$ (the lower limit given by radial velocities and proper motions in the jet, López-Martín et al. 2003). The dashed curves indicate velocities a factor of 2 higher and lower than this mean estimate.

(4) The emission is optically thick in ${ }^{12} \mathrm{CO}$ : As shown in Fig. 4 , the ${ }^{12} \operatorname{CO}(J=2-1) /(J=1-0)$ flux ratio is close to 4 , the square of the frequency ratio $^{3}$, as expected for optically thick emission in the Rayleigh-Jeans limit $\left(B_{v}(T) \simeq 2 k T v^{2} / c^{2}\right)$.

(5) The integrated ${ }^{12} \mathrm{CO}(J=2-1)$ line profile (see Fig. 4$)$ is double peaked with symmetric wings on either side of the stellar velocity. It bears a strong resemblance with the characteristic profile shape of rotating disks around CTTS, as observed e.g. in

\footnotetext{
${ }^{3}$ A ratio slightly less than 4 is found toward the red peak of the line profile, due to slight contamination by the red-shifted "arm" in the larger ${ }^{12} \mathrm{CO}(J=1-0)$ beam.
} 


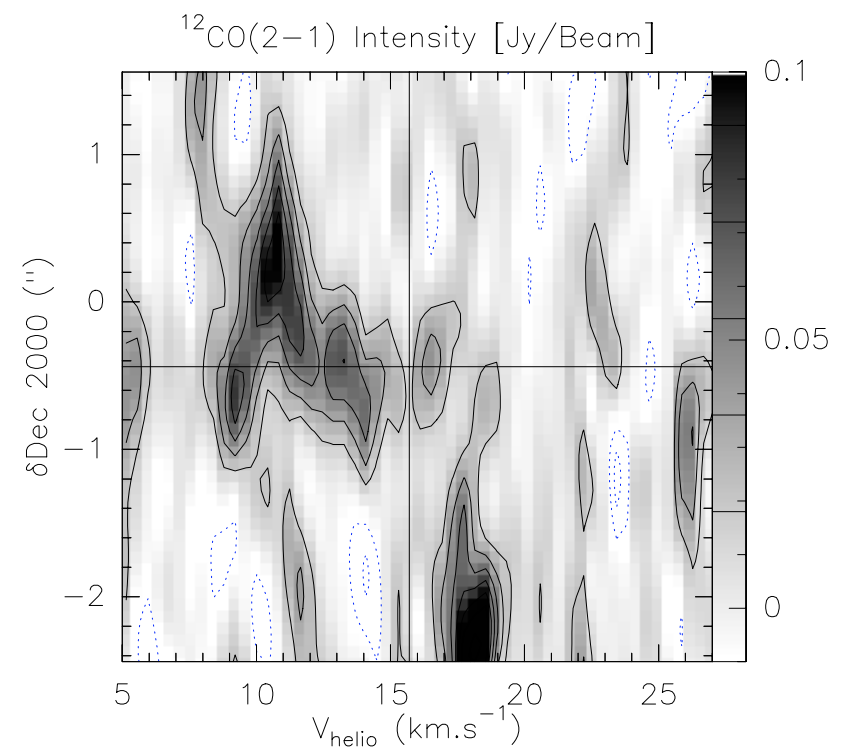

Fig. 5. ${ }^{12} \mathrm{CO} J=2-1$ position-velocity diagram in the North-South direction through RW Aur B, illustrating the asymmetry in velocity and spatial extension of the associated molecular peak. The horizontal line shows the declination of RW Aur B. The vertical line indicates the RW Aur A average systemic velocity. The bright region at $\left(19 \mathrm{~km} \mathrm{~s}^{-1},-2.4^{\prime \prime}\right)$ is the redshifted arm. The data cube was smoothed by a $1^{\prime \prime}$ Gaussian in RA to increase the $\mathrm{S} / \mathrm{N}$ ratio. Contour spacing is $18 \mathrm{mJy} /$ beam ( $1.5 \sigma$ in the smoothed cube).

LkCa 15 and DM Tau (Duvert et al. 2000; Guilloteau \& Dutrey 1994).

All of the above facts strongly argue that the $\mathrm{CO}$ emission associated with RW Aur A is tracing an optically thick disk perpendicular to the jet axis, with its rotation vector $\boldsymbol{\Omega}$ pointing in the direction of the redshifted jet. Physical disk parameters (size, temperature, mass) are estimated in Sect. 4.1 from models of the line profiles.

\subsubsection{RW Aur B molecular peak}

The weaker molecular peak found towards RW Aur B is only detected in ${ }^{12} \mathrm{CO}(J=2-1)$. Its centroid is markedly blue-shifted with respect to RW Aur A (Fig. 1). Inspection of the channel maps shows that emission covers a broad velocity range $V_{\text {helio }} \simeq 9-15 \mathrm{~km} \mathrm{~s}^{-1}$ and has a complex, asymmetric spatial and velocity structure. This asymmetry contrasts markedly with the nicely symmetrical pattern observed in the molecular peak around RW Aur A (see above). It is particularly evident in a North-South PV diagram through RW Aur B, presented in Fig. 5.

To the north of RW Aur B, one observes a spatially extended "lobe" sharply peaked in velocity at $V_{\text {helio }} \simeq 11 \mathrm{~km} \mathrm{~s}^{-1}$. Channel maps indicate a size of $1^{\prime \prime}(\mathrm{N}-\mathrm{S})$ by $0.5^{\prime \prime}(\mathrm{E}-\mathrm{W})$ for this lobe. To the south of RW Aur B, emission is much more spatially compact but splits into two distinct velocity components: A narrow one at $\simeq 9 \mathrm{~km} \mathrm{~s}^{-1}$, centered $0.2^{\prime \prime}$ south of RW Aur B, and a broader one at $\simeq 12.5-14.5 \mathrm{~km} \mathrm{~s}^{-1}$, that appears to describe a clockwise spiral pattern around RW Aur B in the channel maps. Both of these velocity components connect smoothly to the northern lobe in the PV diagram.

In addition to these main structures, the PV diagram shows a $3 \sigma$ peak at $16.1-16.5 \mathrm{~km} \mathrm{~s}^{-1}$ towards RW Aur B. It apparently corresponds to a faint bridge of emission between RW Aur A and RW Aur B in the channel maps.

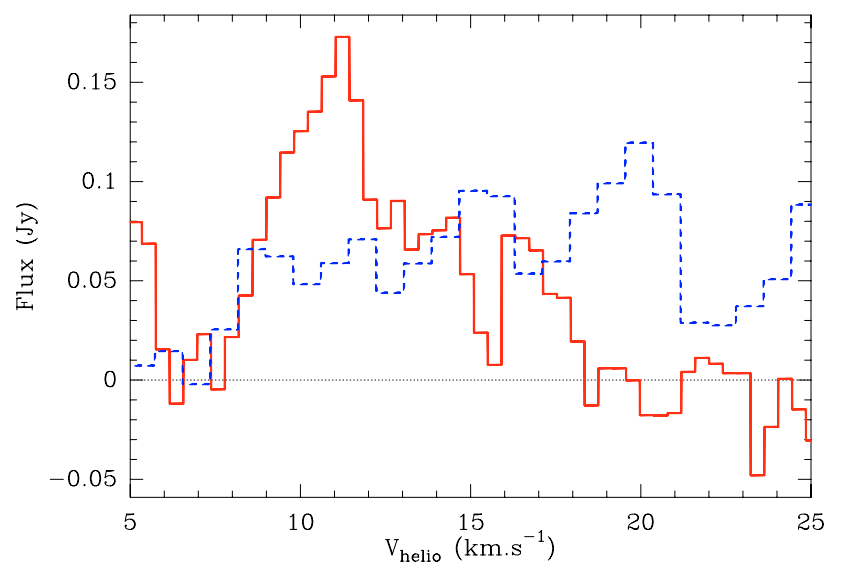

Fig. 6. ${ }^{12} \mathrm{CO}$ spectra towards RW Aur B. Continuum emission is subtracted. The solid histogram displays the $J=2-1$ spectrum, integrated over the $J=1-0$ beam $\left(2.42^{\prime \prime} \times 1.55^{\prime \prime}\right.$ at PA $\left.=24^{\circ}\right)$. The dashed histogram displays the $J=1-0$ spectrum, multiplied by 4 (the $3 \sigma$ level is then $0.12 \mathrm{Jy}$ ). The emission component at $11 \mathrm{~km} \mathrm{~s}^{-1}$ appears partly optically thin.

Given the presence of two velocity components to the south of RW Aur B, and the marked North-South asymmetry, it is not possible to identify an unambiguous rotating disk signature in the data. Based on the PV diagram, the systemic velocity of RW Aur B could be either $V_{\text {helio }} \simeq 10 \mathrm{~km} \mathrm{~s}^{-1}$ or $V_{\text {helio }} \simeq 13 \mathrm{~km} \mathrm{~s}^{-1}$. In either case, RW Aur B is blueshifted with respect to RW Aur A by $\simeq 3-6 \mathrm{~km} \mathrm{~s}^{-1}$, and the kinematics of circumstellar material must include highly disturbed motions in addition to any rotation.

As shown in Fig. 6, no ${ }^{12} \mathrm{CO}(J=1-0)$ emission is firmly detected toward RW Aur B. The $3 \sigma$ upper limit of $30 \mathrm{mJy}$ yields a flux ratio $(J=2-1) /(J=1-0) \geq 5.7$ at $11 \mathrm{~km} \mathrm{~s}^{-1}$, indicating marginally optically thin emission in the extended northern lobe that emits at this velocity. The upper limit in ${ }^{12} \mathrm{CO}(J=1-0)$ does not constrain the optical depth in the fainter 9 and $13 \mathrm{~km} \mathrm{~s}^{-1}$ components. The integrated ${ }^{12} \mathrm{CO}(J=2-1)$ flux of $0.59 \mathrm{Jy} \mathrm{km} \mathrm{s}^{-1}$ (Table 4) yields a minimum gas mass of $\simeq 10^{-6}(T / 50 \mathrm{~K}) M_{\odot}$, assuming optically thin emission in LTE at temperature $T \geq 10 \mathrm{~K}$ and a standard $\mathrm{CO}$ abundance.

\subsubsection{The red-shifted arm}

The large reshifted "arm" wrapping around RW Aur A, of total length $\simeq 4$ " $=600 \mathrm{AU}$, is the most unusual feature in our map. Unlike the circumbinary molecular rings seen in GG Tau and UY Aur (Guilloteau et al. 1999; Duvert et al. 1998), the arm exhibits kinematics incompatible with pure rotation motions: rotation would produce a gradient from blue to redshifted emission along the major axis of the arm (i.e. from N-E to S-W). Instead, the largest red-shift is found midway along the arm, and velocities become bluer towards both the N-E and the S-W tips (cf. the centroid map in Fig. 1c). This behavior implies a dominant component of infall or expansion.

Two observational facts favor expansion motions over infall:

(1) The maximum centroid velocity in the arm, $V_{\text {helio }}=$ $+19.5 \mathrm{~km} \mathrm{~s}^{-1}$, corresponds to a redshift of $\simeq+3.8 \mathrm{~km} \mathrm{~s}^{-1}$ with respect to RW Aur A and $\geq 6 \mathrm{~km} \mathrm{~s}^{-1}$ with respect to RW Aur B. The escape velocity at the corresponding distance $\left(1.7^{\prime \prime}=240 \mathrm{AU}\right)$ is $3.2 \mathrm{~km} \mathrm{~s}^{-1}$ from RW Aur A and $2.6 \mathrm{~km} \mathrm{~s}^{-1}$ from RW Aur B (masses of 1.4 and $0.9 M_{\odot}$ respectively (Ghez et al. 1997)). Since the measured redshift 


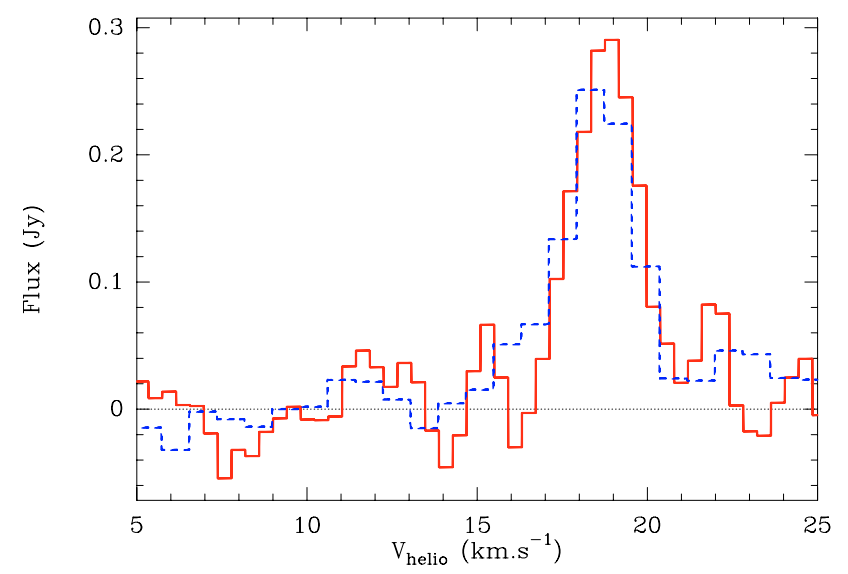

Fig. 7. ${ }^{12} \mathrm{CO}$ spectra towards the redshifted arm, centered at offset $\left(-0.95^{\prime \prime},-2.25\right)$ to avoid confusion from RW Aur A. Continuum emission is subtracted. The solid histogram displays the $J=2-1$ spectrum, integrated over the $J=1-0$ beam $\left(2.42^{\prime \prime} \times 1.55^{\prime \prime}\right.$ at $\left.\mathrm{PA}=24^{\circ}\right)$. The dashed histogram displays the $J=1-0$ spectrum, multiplied by 4 . The emission appears optically thick.

and distance are only lower limits (uncorrected for inclination to the line of sight), material in the red-shifted arm appears at least partly unbound.

(2) If the arm lies roughly in the same plane as the RW Aur A disk (as expected from angular momentum considerations), the fact that the South-East jet lobe points toward us implies that the arm lies on the far side of the RW Aur A disk. Its redshift then implies that it is expanding away from RW Aur A.

We note that the arm appears connected to the N-E of RW Aur A by faint blueshifted emission, as can be seen in the maps of Fig. 1b,c and individual channel maps between 12.8 and $16 \mathrm{~km} \mathrm{~s}^{-1}$. Given the rotation sense of the RW Aur A disk, the morphology of the arm is suggestive of a trailing spiral.

The ${ }^{12} \mathrm{CO}(J=2-1)$ and ${ }^{12} \mathrm{CO}(J=1-0)$ line profiles in the arm are compared in Fig. 7, where the ${ }^{12} \mathrm{CO}(J=2-1)$ flux was integrated over the beam size of the $2.6 \mathrm{~mm}$ observations. The beam was centered at $\left(-0.95^{\prime \prime},-2.25^{\prime \prime}\right)$, to minimize contribution from the strong RW Aur A peak. The $(J=2-1) /(J=1-0)$ line ratio is compatible with the value of 4 for optically thick emission. The typical arm surface brightness of $0.2 \mathrm{Jy} / \mathrm{beam}$ in channel maps then yields an excitation temperature of about $10 \mathrm{~K}$ (as the arm is well resolved, there is no need to correct for beam dilution). The integrated ${ }^{12} \mathrm{CO}(J=2-1)$ flux of $3.7 \mathrm{Jy} \mathrm{km} \mathrm{s}^{-1}$ (Table 4) gives a mass in the arm of $5 \times 10^{-6} \times$ $\tau_{12} /\left(1-\exp \left(-\tau_{12}\right)\right) M_{\odot}$, assuming a standard $\mathrm{CO}$ abundance and LTE at $10 \mathrm{~K}$, where $\tau_{12}$ is the ${ }^{12} \mathrm{CO}(J=2-1)$ optical depth.

\section{Discussion}

\subsection{Properties of the RW Aur A disk}

We do not attempt to carry out a detailed $\chi^{2}$ fit of the CO disk parameters in the $u v$-plane, since the disk is not sufficiently well resolved and other emission structures present in our field of view - the "arm" and RW Aur B peak - would introduce unknown uncertainties and artifacts. As shown below, we may still obtain rough estimates of several key parameters of the RW Aur A disk from the integrated optically thick ${ }^{12} \mathrm{CO}$ line profile, following the modelling results of Beckwith \& Sargent (1993, hereafter BS93).

The inferred parameters will depend explicitly on the adopted mass $M_{\star}$ for RW Aur A. This is not a major
Table 5. Parameters of the RW Aur A disk inferred from comparison with models of Keplerian disks with $T(r) \propto r^{-0.5}$ (see Sect. 4.1). The $i=45^{\circ}$ case is favored. Listed values are for $M_{\star}=1.4 M_{\odot}$ and $d=$ $140 \mathrm{pc}$.

\begin{tabular}{lrrl}
\hline \hline Parameter & $i=45^{\circ}$ & $i=60^{\circ}$ & Scalings \\
\hline$R_{\text {out }}(\mathrm{AU})$ & $41 \pm 2$ & $57 \pm 2$ & $\propto M_{\star}$ \\
$T_{\text {out }}(\mathrm{K})$ & $107 \pm 5$ & $60 \pm 5$ & $\propto\left(d / M_{\star}\right)^{4 / 3}$ \\
$N_{\text {warm }}$ & $\geq 0.5$ & $\geq 0.12$ & $\propto\left(d / M_{\star}\right)^{10 / 3}$ \\
$\quad\left(10^{22} \mathrm{~cm}^{-2}\right)$ & $\leq 10$ & $\leq 2.4$ & \\
$M_{\text {warm }}$ & $\geq 0.13$ & $\geq 0.06$ & $\propto d^{10 / 3} M_{\star}^{-4 / 3}$ \\
$\quad\left(10^{-4} M_{\odot}\right)$ & $\leq 2.7$ & $\leq 1.1$ & \\
\hline
\end{tabular}

uncertainty, however ${ }^{4}$. In the following we will adopt $M_{\star}=$ $1.4 M_{\odot}$ as our nominal value. The scalings of disk parameters with $M_{\star}$ (and distance) are given in Table 5 .

\subsubsection{Disk velocity law and inclination}

The RW Aur A disk is not sufficiently well resolved with PdBI to enable a direct determination of its rotation law. However, it is expected that gas dissipation will rapidly re-circularize disk motions, even after substantial tidal disturbance (Clarke \& Pringle 1993), hence rotation is likely to be close to Keplerian over most of the disk.

BS93 demonstrate that the integrated line profile from an optically thick Keplerian disk has power-law wings with $I(v) \propto$ $v^{3 q-5}$, where $q$ is the index of the radial distribution of gas temperature, $T(r) \propto r^{-q}$. We find that the $\mathrm{CO}(2-1)$ line profile of the RW Aur A disk (Fig. 4) indeed exhibits symmetric power-law wings, of slope $\simeq-2.8$. This would be consistent with Keplerian rotation provided the $\mathrm{CO}$ photosphere has $q \simeq 0.73$, only slightly steeper than the dust temperature law inferred from the infrared SED (Osterloh \& Beckwith 1995, $q \simeq 0.57$ ).

We will thus use the modelled profiles presented by BS93 for Keplerian disks with $q=0.5$ to obtain rough first-order estimates of the overall disk properties in RW Aur A, keeping in mind that higher angular resolution observations will be needed for more accurate determinations. Another source of uncertainty in this comparison comes from the simplifying assumption in the BS93 models of a vertically isothermal disk, which may not be fully valid here, given the high gas temperatures that are inferred (see below).

Inferred disk parameters will slightly depend on the adopted disk inclination $i$ to the line of sight. The ratio of proper motion $V_{\perp}$ to radial velocity $V_{\text {rad }}$ towards emission knots in the RW Aur jet suggests a jet inclination $i=\arctan \left(V_{\perp} / V_{\text {rad }}\right)=$ $46^{\circ} \pm 3^{\circ}$ to the line of sight (López-Martín et al. 2003). Implicit in this estimate is the assumption that knots trace "internal working surfaces" roughly perpendicular to the jet flow, so that the hot postshock gas where $V_{\text {rad }}$ is measured is at rest with respect to the moving knots (Raga et al. 1990). If, on the other hand, the knots traced highly oblique shocks along the jet wall, the emitting postshock gas would have a non-zero tangential motion with respect to the shock wave and the inclination derived from the knot proper motion would be a lower limit only.

In the case of DG Tau, the internal working surface hypothesis yields a jet inclination $i \simeq 45^{\circ}$ (Pyo et al. 2003) in excellent

\footnotetext{
4 Recent studies give consistent estimates $M_{\star} \simeq 1.3-1.4 M_{\odot}$, despite slight differences in adopted spectral type $(\mathrm{K} 1-\mathrm{K} 4)$ and evolutionary tracks, and the use of optical vs near-IR photometry (Ghez et al. 1997; Woitas et al. 2001). The same mass range is obtained with the pms tracks of Siess et al. (2000).
} 
agreement with the stellar spin axis inclination $i=36^{\circ} \pm 4^{\circ}$ inferred from the stellar period, radius, and $v \sin i$ (Bouvier et al. 1993; Doppmann et al. 2005). In the case of RW Aur A, the stellar rotation period and $v \sin i$ are ambiguous (Petrov et al. 2001), preventing a similar test. However, the radial velocities of up to $-180 \mathrm{~km} \mathrm{~s}^{-1}$ in the blue jet (Woitas et al. 2002) indicate that $i$ cannot be much larger than $60^{\circ}$, as deprojected jet speeds would otherwise exceed the maximum wind speeds ever observed in CTTS. We conclude that $i$ lies between $45^{\circ}$ and $60^{\circ}$ in RW Aur A.

\subsubsection{Outer disk radius}

As shown by BS93, the optically thick profile shape at a given inclination depends only on $\left(v / v_{\mathrm{d}}\right)$ where $v_{\mathrm{d}}=\sqrt{G M_{\star} / R_{\text {out }}}$ is the Keplerian velocity at the outer disk radius, $R_{\text {out }}$. In particular, the two peaks in the line profile are located roughly at projected velocities $\pm v_{\mathrm{p}} \simeq \sin i v_{\mathrm{d}}$. With $M_{\star}=1.4 M_{\odot}$, the observed peak location at $v_{\mathrm{p}} \simeq 3.5 \mathrm{~km} \mathrm{~s}^{-1}$ suggests $R_{\text {out }} \simeq 50-77 \mathrm{AU}$ for $i \simeq$ $45-60^{\circ}$.

A more accurate estimate is obtained by detailed comparison of the observed profile with theoretical ones (Fig. 5 of BS93), after stretching the velocity axis by $\sqrt{M_{\star} / R_{\text {out }}}$. We find that the line profile shape is best reproduced by a model at $i=45^{\circ}$ with $R_{\text {out }} \simeq 41 \pm 2$ AU. This model is plotted on top of the observed profile in Fig. 4. An acceptable agreement is also obtained for $i=60^{\circ}$ with $R_{\text {out }} \simeq 57 \pm 2 \mathrm{AU}$, although the central dip is now deeper than observed (lower model curve in Fig. 4). The $i=45^{\circ}$ case thus appears favored.

In either case, the RW Aur A disk is the smallest detected so far in CO around a CTTS (previously reported CO disk radii are in the range $120-800 \mathrm{AU}$ ). Such a small disk radius readily explains the unusual detection of the red-shifted optical jet lobe down to $0.2^{\prime \prime}$ from the star (Woitas et al. 2002).

\subsubsection{Gas temperature: evidence for a warm $\mathrm{CO}$ layer}

BS93 find that the peak intensity of an optically thick line from a Keplerian disk scales as (cf. their Eq. (29))

$$
F_{v}(\text { peak }) \propto \lambda_{\mathrm{mm}}^{-2} R_{\text {out }}{ }^{5 / 2} T_{\text {out }}{ }^{3 / 2} M_{\star}{ }^{-1 / 2} d^{-2},
$$

where $T_{\text {out }}$ is the gas temperature at the outer radius. Since the numerical factor in Eq. (29) of BS93 does not include the effect of inclination, we have applied the above flux scalings directly to the individual ${ }^{13} \mathrm{CO}(J=1-0)$ profiles in Fig. 5 of BS93. We find that the observed ${ }^{12} \mathrm{CO}(J=2-1)$ and $(J=1-0)$ fluxes require $T_{\text {out }}=107 \pm 5 \mathrm{~K}$ for $i=45^{\circ}$ (favored case) and $T_{\text {out }}=60 \pm 5 \mathrm{~K}$ for $i=60^{\circ}$. The scaled profiles are shown in Fig. 4 .

Another way to see the need for a high temperature at $R_{\text {out }}$ is to consider the brightness temperature at the line peaks ( $\simeq 13 \mathrm{~K}$ using the conversion factor in Table 1 ), and correct it for beam dilution; Fig. 3a of BS93 indicates an emitting surface at $v_{\mathrm{p}}$ of about $1 / 4$ of the projected disk surface for $i=45^{\circ}$. Hence one infers an intrinsic emission temperature $T \simeq 13 \times($ Beam area $) /\left(\cos i \pi R_{\text {out }}^{2} / 4\right) \simeq 110 \mathrm{~K}$, close to the value derived above.

The temperature of the $\mathrm{CO}$ emitting layer is significantly higher than the mean dust temperature $T_{\mathrm{d}}$ at the same distance: One expects only $T_{\mathrm{d}} \simeq 30 \mathrm{~K}$ at $40 \mathrm{AU}$ from both stellar illumination and accretion (Chiang \& Goldreich 1997; D'Alessio et al. 1999). A low value of $T_{\mathrm{d}}(40 \mathrm{AU}) \simeq 25 \mathrm{~K}$ is independently indicated by fits to the infrared SED of RW Aur A, which yield $T_{\mathrm{d}}(r)=217 \mathrm{~K}(r / \mathrm{AU})^{-0.57}$ (Osterloh \& Beckwith 1995). Hence, the RW Aur A CO disk provides evidence for a gas temperature increase in the upper disk atmosphere. Indications of increased gas temperature in upper disk layers were already inferred by Dartois et al. (2003) in the context of the DM Tau disk, from comparison of lines from various isotopologues of $\mathrm{CO}$. The RW Aur A observations probe even higher CO temperatures than in DM Tau and other previously studied TTS disks, with the possible exception of BP Tau (where $T(\mathrm{CO}) \simeq 50-80 \mathrm{~K}$ at $50 \mathrm{AU}$; Dutrey et al. 2003). This could result from the unusually small disk radii probed by the present observations, or from the presence of an additional gas heating process (see below).

\subsubsection{Surface density of the warm CO layer}

The fact that the line peaks appear optically thick in ${ }^{12} \mathrm{CO}(J=1-0)$ (see Sect. 3.2.1) but are not detected in ${ }^{13} \mathrm{CO}(J=1-0)$, may be used to constrain the surface density, $\Sigma_{\text {warm }}$, of the warm CO emitting layer near $R_{\text {out }}$. BS93 find that line opacity does not appear to vary much with inclination, with face-one and edge-on disks having nearly the same optical depths (velocity shear roughly compensating for the increased path-length). We may thus obtain a rough estimate of $\Sigma_{\text {warm }}$ using Eq. (12) of BS93 for the pole-on optical depth in ${ }^{13} \mathrm{CO}(J=1-0)$

$\Sigma_{\mathrm{warm}} \simeq \frac{\tau_{13}\left(v_{\mathrm{p}}\right)}{2000}\left(\frac{T_{\mathrm{out}}}{5.29}\right)^{5 / 2}$

where we have used the fact that emission at $\pm v_{\mathrm{p}}$ is dominated by regions near $R_{\text {out }}$. Note that a standard ISM fractional abundance ${ }^{13} \mathrm{CO} / \mathrm{H}_{2}=10^{-6}$ is assumed. Indeed, we expect little $\mathrm{CO}$ depletion on ice mantles in the warm layer of the RW Aur disk, given the high temperature.

We concentrate on the blue-shifted peak of the profile, which is not contaminated by the redshifted arm. There, the upper limit on ${ }^{12} \mathrm{CO}(J=1-0) /{ }^{13} \mathrm{CO}(J=1-0) \simeq 80 / 21$ indicates $\tau_{13}\left(v_{\mathrm{p}}\right)<$ 0.25 . In the favored case of $i=45^{\circ}$, where $T_{\text {out }} \simeq 107 \mathrm{~K}$ (see above) we infer $\Sigma_{\text {warm }} \leq 0.23 \mathrm{~g} \mathrm{~cm}^{-2}$. Similarly, the fact that ${ }^{12} \mathrm{CO}(J=1-0)$ is optically thick imposes $\tau_{13}\left(v_{\mathrm{p}}\right)>0.0125$ (assuming a typical abundance of ${ }^{12} \mathrm{CO} / \mathrm{H}_{2}=8 \times 10^{-5}$ ), hence $\Sigma_{\text {warm }} \geq 0.011 \mathrm{~g} \mathrm{~cm}^{-2}$. The approximate range of surface densities for the warm CO layer at $R_{\text {out }}$ is thus

$0.011 \mathrm{~g} \mathrm{~cm}^{-2} \leq \Sigma_{\text {warm }} \leq 0.23 \mathrm{~g} \mathrm{~cm}^{-2}$,

or, in terms of the column density of $\mathrm{H}$ nuclei, $N_{\text {warm }}$ (after correcting for $40 \%$ of helium in mass),

$5 \times 10^{21} \mathrm{~cm}^{-2} \leq N_{\text {warm }} \leq 10^{23} \mathrm{~cm}^{-2}$.

Assuming a surface density law $\Sigma(r) \propto 1 / r$, the total mass of the warm CO layer, $M_{\text {warm }}=2 \pi R_{\text {out }}^{2} \Sigma_{\text {warm }}$, would lie in the range:

$10^{-5} M_{\odot} \leq M_{\text {warm }} \leq 3 \times 10^{-4} M_{\odot}$.

The surface densities and masses in the case $i=60^{\circ}$ are 2-4 times lower (see Table 5).

Note that our observations do not constrain the amount of mass "hidden" below the ${ }^{12} \mathrm{CO}$ photosphere, in the colder interior probed by the dust continuum. Such a cold disk midplane would remain undetected in ${ }^{13} \mathrm{CO}(J=1-0)$, even if optically thick in that line, due to the intrinsically small disk radius: using Eq. (29) of BS93 one predicts a peak ${ }^{13} \mathrm{CO}(J=1-0)$ line flux of $7 \mathrm{mJy}$ only (i.e. our $1 \sigma$ level) for a temperature of $T_{\text {out }}=$ $T_{\mathrm{d}}=25 \mathrm{~K}$ at $R_{\text {out }}=40 \mathrm{AU}$.

Interestingly, the column density we infer for the warm $\mathrm{CO}$ layer largely exceeds that of the "superheated" dust layer in 
well-mixed models of illuminated flared disks: a visual opacity $\tau_{V} \simeq 1$ along the slanted path to the star implies a vertical column density for the superheated layer $N_{\perp} \ll 10^{21} \mathrm{~cm}^{-2}$ (Chiang \& Goldreich 1997; D’ Alessio et al. 1999). One possibility might be that significant dust settling has occurred, decreasing the visual opacity per unit gas column and moving the superheated dust layer to $N_{\perp} \geq 8 \times 10^{21} \mathrm{~cm}^{-2}$. Evidence for dust settling in the outer regions of CTTS disks was presented e.g. by Miyake \& Nakagawa (1995) based on infrared SED shapes and by Brittain et al. (2005) from CO absorption measurements in the HL Tau disk. A second possibility would be that the gas is heated above the dust equilibrium temperature by strong viscous/turbulent dissipation above the disk midplane (Glassgold et al. 2004). Such enhanced dissipation might be related to the strong tidal interaction suffered by the RW Aur A disk (see next section).

\subsection{The red-shifted arm: a tidal tail}

The redshifted expanding "arm" is an unusual feature not previously observed in a T Tauri system. Based on its connexion to the RW Aur A disk on the side opposite to RW Aur B, its trailing spiral morphology, and its unbound expanding motions (see Sect. 3.2.3), we propose that it is tracing a tidal tail stripped from the RW Aur A disk by the recent fly-by of RW Aur B. Indeed, simulations of an accretion disk response to a stellar fly-by reproduce remarkably well all of the above properties, provided the companion is on a prograde orbit (see e.g. Fig. 2a of Clarke \& Pringle 1993).

In the tidal interaction process, a substantial fraction of the disk mass beyond the truncation radius is ejected in the tidal arm, and a similar fraction is captured by the perturber. The capture process may well be causing the disturbed kinematics and morphology we observe in the RW Aur B molecular peak.

If $\mathrm{B}$ were on a circular orbit around $\mathrm{A}$, the expected tidal truncation limit for the primary disk would be 0.4 of the separation (Papaloizou \& Pringle 1977), i.e. $\geq 80$ AU (the projected $\mathrm{AB}$ angular separation is $1.5^{\prime \prime}=200 \mathrm{AU}$ ). The observed disk radius of only $40-57$ AU thus points to an eccentric orbit for B. The results of Artymowicz \& Lubow (1994) for eccentric systems indicate that the primary disk will be truncated at $R_{\text {out }} \simeq 0.4 p$, where $p=(1-e) a$ is the periastron, for a typical disk viscosity parameter $\alpha=0.1$ and a mass parameter $\mu=M_{2} /\left(M_{1}+M_{2}\right)=0.3$ appropriate to RW Aur. We thus estimate $p \simeq R_{\text {out }} / 0.4=100-140 \mathrm{AU}$.

It is tempting to speculate that the strong tidal interaction we are witnessing might also solve a long standing puzzle, namely how RW Aur A can maintain such a high accretion rate, despite a small disk mass and the lack of ambient cloud reservoir. Indeed, SPH simulations have suggested that dissipation associated with tidal stripping could trigger a transient accretion outburst onto the stars (Bonnell \& Bastien 1992). Then, the current accretion rate in RW Aur A would not be representative of the mean level, and the disk lifetime would be much longer than previously thought. Specific conditions need to be met, however, to prevent the tidally-induced density and bending waves from damping before reaching the inner disk (see e.g. Terquem 2000, and references therein).

\subsection{MHD disk winds in RW Aur}

An important result of our observations concerning the issue of jet launching is that the rotation sense of the Keplerian disk around RW Aur A is opposite to that inferred for its optical jet by Woitas et al. (2005): the latter authors find that "the sense of rotation [of the jet] is anti-clockwise looking from the tip of the blue lobe down to the star". As can be seen in Fig. 3, the $\mathrm{CO}$ disk rotates in the clockwise direction when viewed from the tip of the blue lobe (in other words, the rotation vector $\boldsymbol{\Omega}$ points towards the red-shifted jet lobe). As argued above, we are confident that the observed $\mathrm{CO}$ disk velocity gradient is reliable and is not an artifact, since (1) the data quality is excellent, (2) the gradient direction is exactly perpendicular to the jet axis (Fig. 1c), an unambiguous sign of disk rotation, (3) the emission is point-symmetric in space and velocity with respect to RW Aur A (Fig. 3).

MHD disk winds, where enthalpy is negligible in matter acceleration, are necessarily rotating in the same sense as the underlying disk at the launch point (Anderson et al. 2003, their Eq. (4)). Therefore this discrepancy is quite puzzling. Even though the RW Aur A disk is clearly tidally disturbed, it is very difficult to envision a process that would make it counterrotate with respect to inner disk regions (from where the jet originates). In particular, models of disk dynamics in eccentric systems show that the orbit of RW Aur B must be prograde to produce the strong tidal arm that is observed (Clarke \& Pringle 1993). Therefore, the fly-by of RW Aur B cannot have reverted the rotation sense of the outer disk with respect to the inner disk.

At this stage, the simplest explanation would appear to be that the transverse centroid velocity shifts currently measured in the RW Aur optical jet are dominated by other effects than jet rotation. For example, recent simulations show that a precessing, time-variable jet produces transverse shifts that may mimic jet rotation (Cerqueira et al. 2006). The jet rotation speeds and the launch radii of 0.5-1.6 AU inferred by Woitas et al. (2005) would then be upper limits only.

We stress that this does not rule out altogether an MHD disk wind origin for the optical jet of RW Aur A: the theoretical model of disk wind that best fits the DG Tau jet rotation data, with a magnetic lever arm parameter $\lambda \simeq 13$ (Pesenti et al. 2004), predicts rotation velocities for the RW Aur jet that lie below these upper limits (Ferreira et al. 2006). Optical observations at higher spatial and spectral resolution, probing more axial jet regions, are needed to fully test the disk wind model in this object.

Another result of our observations related to disk winds is the lack of a detectable molecular counterpart to the RW Aur A atomic jet. Scaling from the DG Tau jet (Pesenti et al. 2004) one would expect material launched from disk radii of 1 to $40 \mathrm{AU}$ to reach poloidal speeds in the range $V_{\mathrm{p}} \simeq 80-12 \mathrm{~km} \mathrm{~s}^{-1}$ within $50 \mathrm{AU}$ of the star. No signal was found in this velocity range, even in $3.5 \mathrm{~km} \mathrm{~s}^{-1}$ wide channels, down to $18 \mathrm{mJy}(3 \sigma)$. This limit corresponds to a gas mass per unit velocity of $\mathrm{d} M / \mathrm{d} v \simeq 2.7 \times 10^{-8}(T / 50 \mathrm{~K}) M_{\odot}$ per km s${ }^{-1}$ (assuming a standard CO abundance and LTE).

Self-similar MHD disk wind models predict that in each jet lobe $\mathrm{d} M / \mathrm{d} v=\xi \dot{M}_{\mathrm{acc}} R_{\mathrm{obs}} /\left|V_{\mathrm{p}}\right|^{2}$, where $\xi=\mathrm{d} \log \dot{M}_{\mathrm{acc}} / \mathrm{d} \log R$ is the ejection index and $R_{\mathrm{obs}}$ is the beam radius (centered on the star). Taking $\dot{M}_{\text {acc }}=10^{-6} M_{\odot} \mathrm{yr}^{-1}, R_{\text {obs }}=0.36^{\prime \prime}=$ $50 \mathrm{AU}$, and $\xi=0.04$ (Pesenti et al. 2004), we infer $\mathrm{d} M / \mathrm{d} v=$ $10^{-7}\left(V_{\mathrm{p}} / 10 \mathrm{~km} \mathrm{~s}^{-1}\right)^{-2} M_{\odot}$ per $\mathrm{km} \mathrm{s}^{-1}$. Thus, our non detection in $\mathrm{CO}$ does not rule out molecular MHD ejection from disk radii beyond $1 \mathrm{AU}$ if the molecular jet is hotter than $200 \mathrm{~K}\left(V_{\mathrm{p}} / 10 \mathrm{~km} \mathrm{~s}^{-1}\right)^{-2}$ (e.g. due to X-rays or ambipolar diffusion heating). Rotational or rovibrational $\mathrm{H}_{2}$ emission would then be a better tracer. 


\section{Summary}

Our main results and implications are the following:

- RW Aur A is surrounded by an optically thick CO structure in bound rotation about the optical jet axis. Assuming a Keplerian law, we infer an outer radius $R_{\text {out }} \simeq 40-57 \mathrm{AU}$, the smallest detected so far around a CTTS, and an unusually warm $\mathrm{CO}$ temperature $\left(T_{\text {out }} \simeq 60-100 \mathrm{~K}\right)$. The column density of warm emitting gas is substantial, $N_{\text {warm }} \simeq 0.1-10 \times 10^{22} \mathrm{~cm}^{-2}$, requiring either dust settling towards the midplane (if heating is dominated by stellar illumination) or strong viscous/turbulent dissipation above the disk surface, possibly related to tidal interaction.

- An expanding, partly unbound arm of molecular material is discovered trailing from the RW Aur A disk. We propose that this arm is tracing a tidal tail stripped from the disk by the recent fly-by of RW Aur B. The large arm extent (about $600 \mathrm{AU}$ ) and the (current) radius of the primary disk indicate a prograde fly-by, with periastron $\simeq 100-140 \mathrm{AU}$.

- RW Aur B is blueshifted by $3-6 \mathrm{~km} \mathrm{~s}^{-1}$ with respect to RW Aur A. Its molecular environment is complex and asymmetric, presumably due to tidal interaction and capture of material from the A disk.

- As originally proposed by Bonnell \& Bastien (1992) in the context of FU Ori outbursts, it is tempting to speculate that enhanced dissipation caused by tidal interaction might be responsible for the unusually high accretion rate currently observed in RW Aur A, thus possibly solving the puzzle of its short disk lifetime (1000 yrs). Specific conditions should be present, however, to prevent tidally excited waves from damping before reaching the inner disk (see Terquem 2000, and references therein).

- The rotation sense of the RW Aur A disk is opposite to the jet rotation sense determined by Woitas et al. (2005) from HST/STIS spectro-imaging. The simplest explanation for this discrepancy would be that transverse velocity shifts in the optical jet are dominated by other effects than rotation. The jet rotation speeds and launch radii of 0.5-1.6 AU inferred by Woitas et al. (2005) would then be upper limits only. Such upper limits are still consistent with current models of MHD ejection from the disk (Ferreira et al. 2006).

Acknowledgements. We acknowledge the IRAM staff at Plateau de Bure and Grenoble for carrying out the observations and for the help provided during the data reduction. We also thank I. Bonnell, F. Gueth, F. Ménard, C. Terquem and an anonymous referee for helpful comments on this paper, and A. Dutrey and V. Piétu for the communication of their flux measurements. This research is supported by the JETSET Marie Curie Research and Training Network as part of the European Community's Human Potential Programme, under contract MRTNCT-2004-005592.

\section{References}

Anderson, J. M., Li, Z.-Y., Krasnopolsky, R., \& Blandford, R. D. 2003, ApJ, 590, L107

Andrews, S. M., \& Williams, J. P. 2005, ApJ, 631, 1134

Artymowicz, P., \& Lubow, S. H. 1994, ApJ, 421, 651

Bacciotti, F., Ray, T. P., Mundt, R., Eislöffel, J., \& Solf, J. 2002, ApJ, 576, 222

Basri, G., \& Bertout, C. 1989, ApJ, 341, 340

Beckwith, S. V. W., \& Sargent, A. I. 1993, ApJ, 402, 280

Bertout, C., Robichon, N., \& Arenou, F. 1999, A\&A, 352, 574

Bonnell, I., \& Bastien, P. 1992, ApJ, 401, L31

Bouvier, J., Cabrit, S., Fernandez, M., Martin, E. L., \& Matthews, J. M. 1993, A\&A, 272, 176

Brittain, S. D., Rettig, T. W., Simon, T., \& Kulesa, C. 2005, ApJ, 626, 283

Cerqueira, A. H., Velazquez, P. F., Raga, A. C., Vasconcelos, M. J., \& De Colle, F. 2006, A\&A, 448, 231

Chiang, E. I., \& Goldreich, P. 1997, ApJ, 490, 368

Clarke, C. J., \& Pringle, J. E. 1993, MNRAS, 261, 190

Coffey, D., Bacciotti, F., Woitas, J., Ray, T. P., \& Eislöffel, J. 2004, ApJ, 604, 758

D’Alessio, P., Calvet, N., Hartmann, L., Lizano, S., \& Cantó, J. 1999, ApJ, 527, 893

Dartois, E., Dutrey, A., \& Guilloteau, S. 2003, A\&A, 399, 773

Doppmann, G. W., Greene, T. P., Covey, K. R., \& Lada, C. J. 2005, AJ, 130, 1145

Dougados, C., Cabrit, S., Lavalley, C., \& Ménard, F. 2000, A\&A, 357, L61

Duchêne, G., Monin, J.-L., Bouvier, J., \& Ménard, F. 1999, A\&A, 351, 954

Dutrey, A., Guilloteau, S., \& Simon, M. 2003, A\&A, 402, 1003

Duvert, G., Dutrey, A., Guilloteau, S., et al. 1998, A\&A, 332, 867

Duvert, G., Guilloteau, S., Ménard, F., Simon, M., \& Dutrey, A. 2000, A\&A, 355,165

Ferreira, J., Dougados, C., \& Cabrit, S. 2006, A\&A, in press

Gahm, G. F., Petrov, P. P., Duemmler, R., Gameiro, J. F., \& Lago, M. T. V. T. 1999, A\&A, 352, L95

Ghez, A. M., Neugebauer, G., \& Matthews, K. 1993, AJ, 106, 2005

Ghez, A. M., White, R. J., \& Simon, M. 1997, ApJ, 490, 353

Glassgold, A. E., Najita, J., \& Igea, J. 2004, ApJ, 615, 972

Guilloteau, S., \& Dutrey, A. 1994, A\&A, 291, L23

Guilloteau, S., Dutrey, A., \& Simon, M. 1999, A\&A, 348, 570

Hartigan, P., Edwards, S., \& Ghandour, L. 1995, ApJ, 452, 736

Herbig, G. H., \& Bell, K. R. 1988, Catalog of emission line stars of the orion population: 3: 1988, Lick Observatory Bulletin, Santa Cruz: Lick Observatory Hirth, G. A., Mundt, R., Solf, J., \& Ray, T. P. 1994, ApJ, 427, L99

López-Martín, L., Cabrit, S., \& Dougados, C. 2003, A\&A, 405, L1

Miyake, K., \& Nakagawa, Y. 1995, ApJ, 441, 361

Mundt, R., \& Eislöffel, J. 1998, AJ, 116, 860

Osterloh, M., \& Beckwith, S. V. W. 1995, ApJ, 439, 288

Papaloizou, J., \& Pringle, J. E. 1977, MNRAS, 181, 441

Pesenti, N., Dougados, C., Cabrit, S., et al. 2004, A\&A, 416, L9

Petrov, P. P., Gahm, G. F., Gameiro, J. F., et al. 2001, A\&A, 369, 993

Pyo, T.-S., Kobayashi, N., Hayashi, M., et al. 2003, ApJ, 590, 340

Raga, A. C., Binette, L., Canto, J., \& Calvet, N. 1990, ApJ, 364, 601

Reid, M. J., Schneps, M. H., Moran, J. M., et al. 1988, ApJ, 330, 809

Safier, P. N. 1993, ApJ, 408, 115

Siess, L., Dufour, E., \& Forestini, M. 2000, A\&A, 358, 593

Stout-Batalha, N. M., Batalha, C. C., \& Basri, G. S. 2000, ApJ, 532, 474

Terquem, C. E. J. M. L. J. 2000, The Formation of Binary Stars, ed. R. D. Mathieu, \& H. Zinnecker, ASP Conf. Ser.

Testi, L., Bacciotti, F., Sargent, A. I., Ray, T. P., \& Eislöffel, J. 2002, A\&A, 394, L31

Ungerechts, H., \& Thaddeus, P. 1987, ApJS, 63, 645

White, R. J., \& Ghez, A. M. 2001, ApJ, 556, 265

Woitas, J., Leinert, C., \& Köhler, R. 2001, A\&A, 376, 982

Woitas, J., Ray, T. P., Bacciotti, F., Davis, C. J., \& Eislöffel, J. 2002, ApJ, 580, 336

Woitas, J., Bacciotti, F., Ray, T. P., et al. 2005, A\&A, 432, 149 
S. Cabrit et al.: Tidal stripping and disk kinematics in the RW Aurigae system, Online Material $p 1$

\section{Online Material}


S. Cabrit et al.: Tidal stripping and disk kinematics in the RW Aurigae system, Online Material $p 2$

\section{Appendix A: ${ }^{12} \mathrm{CO}(\mathrm{J}=1-0)$ channel maps}

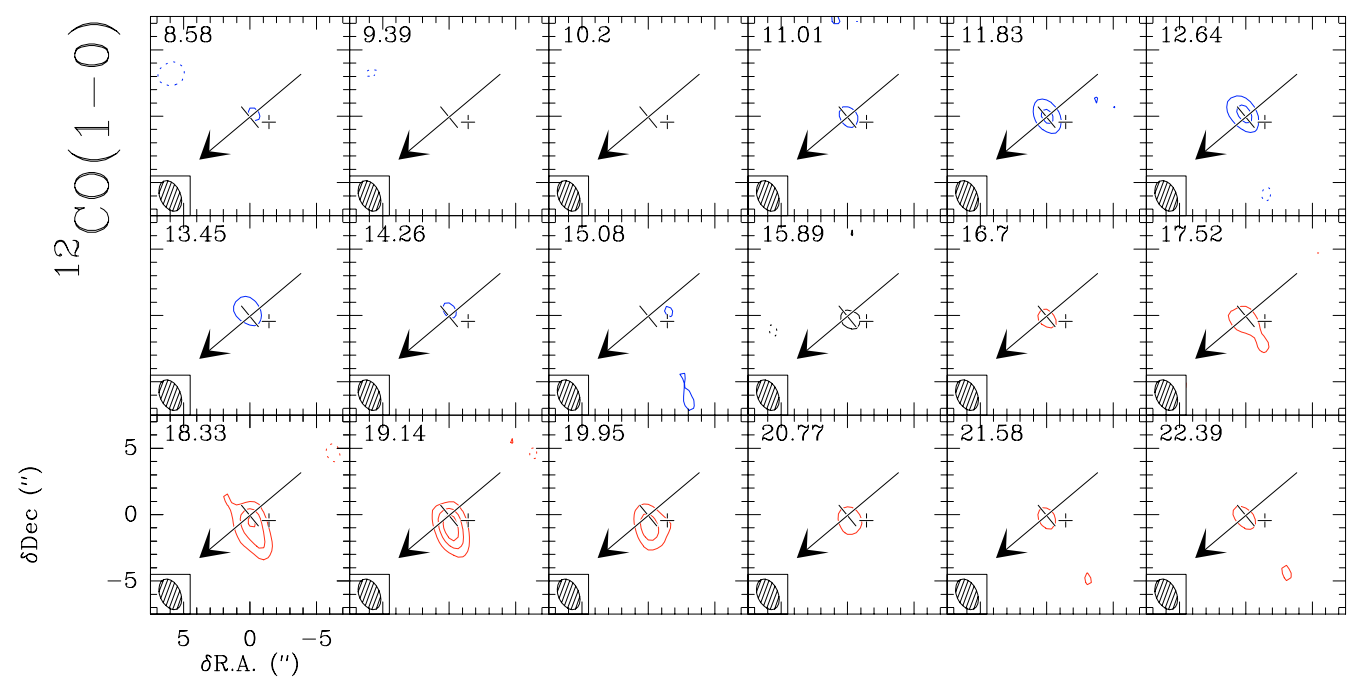

Fig. A.1. Channel maps of ${ }^{12} \mathrm{CO} J=1-0$ towards RW Aur, rebinned to a spectral resolution of $0.85 \mathrm{~km} \mathrm{~s}^{-1}$. Radial velocities (heliocentric) are given in the upper left corner of each panel. The large cross indicates the position of RW Aur A, with an arrow pointing in the blue jet direction. The small cross indicates the position of RW Aur B. Blue and red contours respectively indicate blue and red-shifted channels with respect to the RW Aur A mean heliocentric velocity ( $V_{\text {helio }} \simeq 15.87 \pm 0.55 \mathrm{~km} \mathrm{~s}^{-1}$, Gahm et al. (1999)). Contour levels correspond to $-3,3,6$ and $9 \sigma$ with $1 \sigma=10 \mathrm{mJy} /$ Beam $=0.24 \mathrm{~K}$ (see Table 1 for details). Dotted lines show negative contours. The hatched ellipse represents the beam $F W H M$ size, $2.42^{\prime \prime} \times 1.55^{\prime \prime}$ at $\mathrm{PA}=24^{\circ}$. Continuum is not subtracted. 